\title{
Oxigenoterapia con cánula nasal de alto flujo en lactantes con infección respiratoria baja aguda. Experiencia en hospitales de la Ciudad de Buenos Aires
}

High-flow nasal cannula oxygen therapy in infants with acute lower respiratory tract infection. An experience in hospitals of the City of Buenos Aires

\author{
Dr. Ezequiel Monteverde ${ }^{a}$ Dra. Analía Fernández ${ }^{b}$, Dr. Fernando Ferrero, \\ Dr. Cristian Barbaro ${ }^{a}$, Dr. Leonardo De Lilloc, Dra. Mariana Lavitola ${ }^{b}$ y \\ Dr. Ariel Golubickid
}

Colaboradores: Héctor Cairoli, Edgardo Checacchi, Gustavo Debaisi, Hernán Gaviño, Emiliano Gigliotti, Patricia Landa, Claudia Meregalli, Pablo Neira, Miriam Peralta, Javier Potasznik, Ana Belén Reiner, Alejandra Retta, Julián Rodríguez Kibrik, Guillermo Schwartz y Claudio Zeltman.

\section{RESUMEN}

Introducción. Durante el invierno, los lactantes con infecciones respiratorias agudas bajas (IRAB) sobrecargan los recursos sanitarios. En la Ciudad Autónoma de Buenos Aires, 35000 niños son asistidos anualmente en hospitales; se interna el 8-10\% en unidades generales, y el 5-12\% de ellos, en unidades de terapia intensiva. En 2017, el Departamento de Salud Materno-Infantil de dicha ciudad incluyó la oxigenoterapia por cánula nasal de alto flujo (CNAF) en el protocolo IRAB en unidades generales de tres hospitales. El objetivo de este trabajo es describir los resultados y explorar potenciales factores relacionados con el fracaso terapéutico.

Métodos. Estudio prospectivo descriptivo que incluyó a lactantes $<18$ meses hospitalizados por IRAB en 3 hospitales (Durand, Elizalde, Gutiérrez), de junio a septiembre de 2017. Todos los niños incapaces de cumplir los objetivos terapéuticos con bajo flujo recibieron CNAF, y se consideró fracaso de soporte el ingreso a Terapia Intensiva.

Resultados. De 522 pacientes hospitalizados por IRAB, el 39,7 \% requirieron CNAF. No se observaron diferencias basales significativas entre pacientes con CNAF y con oxigenoterapia convencional.

Solo el 8,7 \% de los pacientes con CNAF presentaron fracaso. Los pacientes con éxito del soporte mostraron un descenso de la frecuencia respiratoria significativamente mayor y más sostenido en el tiempo que aquellos con fracaso $(\mathrm{p}<0,01)$. No se registraron complicaciones.

Conclusión. La implementación protocolizada de CNAF en unidades generales fue una medida segura. Los pacientes con fracaso del soporte mostraron una menor disminución en la frecuencia respiratoria a lo largo del tratamiento. Palabras clave: bronquiolitis, infecciones del sistema respiratorio, terapia por inhalación de oxígeno, insuficiencia respiratoria. http:/ / dx.doi.org/10.5546/ aap.2019.286

Texto completo en inglés:

http:/ / dx.doi.org/10.5546/ aap.2019.eng.286

Cómo citar: Monteverde E, Fernández A,Ferrero F, Barbaro C, et al. Oxigenoterapia con cánula nasal de alto flujo en lactantes con infección respiratoria baja aguda. Experiencia en hospitales de la Ciudad de Buenos Aires. Arch Argent Pediatr 2019;117(5):286-293.

\section{GLOSARIO}

AF: alto flujo

AVM: asistencia ventilatoria mecánica

BF: bajo flujo

CABA: Ciudad Autónoma de Buenos

Aires

CNAF: cánula nasal de alto flujo

CNAF: cánulas nasales de alto flujo

ECC: enfermedes crónicas complejas

ES: éxito de soporte

FC: frecuencia cardíaca

$\mathrm{FiO}_{2}$ : fracción inspirada de oxígeno

FR: frecuencia respiratoria

FS: fracaso de soporte

HD: Hospital General de Agudos

Carlos Durand

HE: Hopital General de Niños

Pedro de Elizalde

HG: Hospital General de Niños

Ricardo Gutiérrez

IRAB: infecciones respiratorias

agudas bajas

RIC: rango intercuartil

$\mathrm{SaO}_{2}$ : saturación de oxígeno 
UCIP: unidades de cuidados intensivos pediátricos UIGP: unidades de internación general pediátrica VI: ventilación invasiva

VMI: ventilación mecánica invasiva

VNI: ventilación no invasiva

VSR: virus sincicial respiratorio

WRT: test de rangos de Wilcoxon (Wilcoxon rank test)

\section{INTRODUCCIÓN}

Los pacientes pediátricos con infecciones respiratorias agudas bajas (IRAB) incrementan fuertemente la demanda de asistencia durante el período invernal, y la bronquiolitis es la principal entidad clínica. ${ }^{1}$ En la Ciudad Autónoma de Buenos Aires (CABA), alrededor de 35000 pacientes con IRAB son asistidos por el Programa de Internación Abreviada dependiente del sistema de salud pública cada año. ${ }^{2}$ Entre el $8 \%$ y el $10 \%$ de estos pacientes ingresa a unidades de internación general pediátrica (UIGP) y, de ellos, del $5 \%$ al $12 \%$ requiere asistencia ventilatoria mecánica (AVM), lo que representa, entre mayo y septiembre, una ocupación del $40 \%$ de las unidades de cuidados intensivos pediátricos (UCIP). ${ }^{3}$

Uno de los pilares del tratamiento de estos pacientes es la oxigenoterapia, tanto por sistemas de bajo flujo (BF) como de alto flujo (AF). Se considera $\mathrm{AF}$ cuando supera $2 \mathrm{l} / \mathrm{min}$ (neonatos), 4 $1 /$ min (lactantes y niños) y $61 / \mathrm{min}$ (adultos). Las cánulas nasales de alto flujo (CNAF) son sistemas abiertos que permiten entregar AF de oxígeno con temperatura y humedad relativa óptimas. Sus principales mecanismos de acción son el lavado del espacio muerto nasofaríngeo, la provisión de flujo adecuado al incremento de la demanda inspiratoria, la entrega de una fracción inspirada de oxígeno $\left(\mathrm{FiO}_{2}\right)$ medible y la generación de presión positiva continua en la vía aérea. ${ }^{4}$

El uso de las CNAF ha sido descrito en diversos escenarios, en neonatos y niños. Estudios observacionales y experimentales muestran una reducción del ingreso a la UCIP ${ }^{5-9}$ y del requerimiento de $\mathrm{AVM}^{0-12}$ asociada a su uso en pacientes con IRAB. Por ello, además de ser mejor tolerado y menos costoso que la ventilación no invasiva (VNI), el soporte con CNAF resulta cada vez más atractivo. Sin embargo, un escaso número de UIGP lo utiliza.

En 2017, el Departamento de Salud MaternoInfantil del Ministerio de Salud de la CABA incluyó el soporte con CNAF en el tratamiento de los pacientes con IRAB en UIGP de tres hospitales. La decisión se basó en que esta estrategia podría mejorar la evolución de los pacientes, lo que reduciría la proporción que requeriría tratamiento en la UCIP. Para su implementación, se diseñó un protocolo de aplicación específico (véase el material suplementario en el Anexo en formato electrónico). El presente reporte tiene como objetivo describir los resultados de esa implementación y explorar potenciales factores asociados al fracaso terapéutico.

\section{MATERIALES Y MÉTODOS}

Diseño. Estudio multicéntrico, prospectivo y descriptivo.

Ámbitos. Hospital General de Agudos Carlos Durand (HD) (UIGP y UCIP), Hospital General de Niños Pedro de Elizalde (HE) (UIGP 4 y 5, y UCIP) y Hospital General de Niños Ricardo Gutiérrez (HG) (UIGP 7 y 8, y UCIP).

Duración. Del 3/6/2017 al 28/9/2017 (semanas epidemiológicas 22-39).

Población. Lactantes de entre 30 días y 18 meses de vida con diagnóstico de IRAB según las guías de diagnóstico y tratamiento del Ministerio de Salud de la $\mathrm{CABA}^{2}$ y hospitalizados en las UIGP seleccionadas. Se excluyeron de su uso los siguientes: 1) pacientes que, al ingresar, habían presentado enfermedad no controlada o grave (inestabilidad hemodinámica, incapacidad de proteger la vía aérea, claudicación respiratoria) y 2) pacientes con dependencia crónica de oxígeno o con cardiopatías congénitas cianóticas.

Plan de trabajo. Antes de la implementación del protocolo, los miembros del equipo de salud de las UIGP seleccionadas recibieron una capacitación teórico-práctica a cargo de profesionales experimentados, quienes colaboraron en el diseño de la guía de uso de $\mathrm{CNAF}$, incluida en el protocolo del Ministerio antes mencionado. El algoritmo de selección de pacientes fue incluido en estas guías y colocado impreso en un lugar visible en las UIGP y UCIP participantes.

De acuerdo con estas guías, las CNAF se indicaron en caso de fracaso del soporte con oxigenoterapia de BF (máximo de $31 / \mathrm{min}$ ), determinado por lo siguiente:

1. Imposibilidad de lograr saturación de oxígeno $\left(\mathrm{SaO}_{2}\right)$ por oximetría de pulso $\geq 94 \%\left(\mathrm{SaO}_{2}\right.$ objetivo).

2. Presencia de frecuencia cardíaca (FC) y frecuencia respiratoria (FR) superior a los valores establecidos como límite luego de haber iniciado el soporte con oxigenoterapia de BF y tratamiento médico apropiado o en 
algún momento de la evolución. Los valores límite se establecieron por rangos etarios. ${ }^{13}$ Pacientes $\leq 6$ meses: FC $\geq 140 /$ min y FR $\geq 55 /$ min; pacientes $>6$ meses: $\mathrm{FC} \geq 140 / \min \mathrm{y}$ $\mathrm{FR} \geq 45 / \mathrm{min}$.

3. Incremento del trabajo respiratorio durante la evolución según los criterios del equipo tratante.

La oxigenoterapia de AF se administró a través del sistema de AF humidificador Airvo2 ${ }^{\circledR}$ de Fisher \& Paykel Healthcare ${ }^{\circledR}$ con cánula Optiflow ${ }^{\circledR}$ infant (hasta $201 / \mathrm{min}$ ). La programación inicial fue flujo de $21 / \mathrm{kg} / \mathrm{min}$ y $\mathrm{FiO}_{2}$ de 0,9 , que se disminuyó a menos de 0,6 dentro de la primera hora y luego en fracciones de 0,05 de acuerdo con la $\mathrm{SaO}_{2}$ objetivo, y se mantuvo el flujo constante en $21 / \mathrm{kg} / \mathrm{min}$. En caso de no lograr la $\mathrm{SaO}_{2}$ objetivo, se incrementó la $\mathrm{FiO}_{2}$ al nivel previo. La suspensión de la oxigenoterapia por CNAF se realizó luego de mantener la $\mathrm{SaO}_{2}$ objetivo con $\mathrm{FiO}_{2}$ de 0,21 durante, al menos, 4 horas.

Se sugirió que el tratamiento médico se realizara de acuerdo con la mencionada guía, sin incluir recomendaciones sobre la alimentación de los pacientes en $\mathrm{CNAF}$, lo que quedó a criterio del equipo tratante.

Se realizó un registro on-line en el que se ingresaron los siguientes datos de todos los pacientes con IRAB: fecha de ingreso y egreso hospitalario, edad, peso, sexo, motivo de ingreso, presencia de enfermedades crónicas complejas (ECC), ${ }^{14}$ germen identificado, características de la radiografía de tórax al ingresar, según el equipo tratante, clasificada en 4 patrones. De los pacientes en los que se aplicó CNAF: criterio de inicio, fecha y hora de inicio y finalización de CNAF, FC y FR al ingresar y en horas estandarizadas de registro, y complicaciones. De haberlo requerido: fecha y hora de ingreso a la UCIP y tipo de soporte ventilatorio adicional. Se designaron dos coordinadores responsables por centro y, al menos, un responsable de registro por cada unidad de internación.

Se definió como éxito de soporte (ES) todo caso que requirió solo tratamiento con CNAF en la UIGP, y se consideró fracaso de soporte (FS) lo siguiente: 1) el pase a la UCIP o a Terapia Intermedia, 2) el requerimiento de VNI o ventilación mecánica invasiva (VMI).

Consideraciones éticas: El estudio fue evaluado por un comité de ética en investigación y registrado en el Comité Central de Ética en Investigación del Ministerio de Salud-Gobierno de la Ciudad Autónoma de Buenos Aires bajo el número $281 / 17$. No se requirió el consentimiento informado, dado que esta modalidad de tratamiento se consideró estándar de cuidado en las unidades participantes. ${ }^{2}$

Análisis de datos. Las variables numéricas se describieron como media o mediana y desvío estándar o rango intercuartil (RIC), según el ajuste a la normalidad (verificada con el test Kolmogorov-Smirnov). Las variables categóricas se expresaron como recuento y proporción (\%). La comparación entre proporciones se realizó usando el test de chi ${ }^{2}$ con corrección de Yates o el test de Fisher para las variables categóricas, según correspondiera, y las diferencias entre medias o medianas se verificaron con el test $\mathrm{T}$ de Student o el test de rangos de Wilcoxon (Wilcoxon rank test, WRT). La comparación entre más de dos grupos para variables categóricas se realizó con el test de Kruskal-Wallis. En consideración de ciertas controversias recientes ${ }^{15}$ y para los casos que resultaron controvertidos a juicio del equipo de trabajo, se incluyeron enfoques bayesianos ${ }^{16,17}$ y el propuesto por Jamil et al., ${ }^{18}$ para tablas de contingencia, y se consideró una distribución de Poisson. El análisis de datos se realizó con el software libre R para MacIntosh versión 3.3.3 (2017-3-6) "Another canoe".

\section{RESULTADOS \\ Generales}

En el período de registro, ingresaron 522 pacientes con diagnóstico de IRAB, 207 (el $39,7 \%$ ) de los cuales requirieron CNAF y 19 (el $3,6 \%$ ) presentaron FS. Los pacientes internados en estas UIGP representaron el $100 \%$ de los ingresos por esta causa en el HD, el $80 \%$ en el HG y el $57,3 \%$ en el HE. La proporción de sujetos que recibió CNAF tuvo una amplia variación entre UIGP: el $25 \%$ (HD), el $40 \%$ (HE-U4), el $41 \%$ (HG-U7), el $42 \%$ (HE-U5) y el $45 \%$ (HG-U8). No se observaron diferencias significativas entre los pacientes que tuvieron éxito con $\mathrm{BF}$ y los que requirieron CNAF relacionadas con la edad, el peso, el sexo, el motivo de ingreso o la presencia de ECC. Si bien la proporción de pacientes con cardiopatías congénitas que requirió CNAF duplicó la que solo recibió BF (el $6 \%$ vs. el $3 \%$ ), esta diferencia no fue estadísticamente significativa. Los que requirieron $\mathrm{CNAF}$ tuvieron más identificación de gérmenes (el $57 \%$ vs. el $40 \%$ ), y el virus sincicial respiratorio (VSR) fue el más frecuente. Menor porcentaje de pacientes con radiografía de tórax normal al ingresar requirió CNAF (el $3 \%$ vs. el $11 \%, \mathrm{p}<0,01$ ) (Tabla 1). Algunas características basales fueron diferentes entre los tres hospitales (Tabla 2). 


\section{Características de la población con cánula nasal de alto flujo}

Todos los pacientes a los que se les indicó CNAF cumplieron con, al menos, un criterio de inicio de CNAF establecido en las guías (Tabla 3), y más del $90 \%$ cumplió con, al menos, uno de los dos primeros.
No se observaron diferencias estadísticamente significativas en el tiempo transcurrido desde el ingreso al inicio de CNAF entre los grupos de ES y FS: 2 horas $(0,5-14,1)$ vs. 0,9 horas $(0,5-4,3)$, $\mathrm{p}=0,175(W R T)$. El tiempo medio trascurrido desde el ingreso hasta el FS fue de 20,6 horas (1384). La duración del soporte con CNAF fue de 98,3 horas (66-147) en aquellos con ES.

TABLA 1. Características basales de los pacientes con y sin cánula nasal de alto flujo

\begin{tabular}{|c|c|c|c|}
\hline & Sin CNAF $(n=315)$ & Con $\operatorname{CNAF}(n=207)$ & $p$ \\
\hline Edad (meses), med. (RIC) & $6,0(3,0-11,0)$ & $6,0(3,0-10,0)$ & NS \\
\hline Sexo masculino, n (\%) & $194(62)$ & $123(59)$ & NS \\
\hline Peso (kg), med. (RIC) & $7,0(5,9-9,0)$ & $7,0(6,0-8,8)$ & NS \\
\hline Puntaje $Z^{*}$ de peso (puntos), media $+/-D E$ & $-0,96 \pm 1,76$ & $-1,06 \pm 1,60$ & NS \\
\hline \multicolumn{4}{|l|}{ Motivo de ingreso } \\
\hline Bronquiolitis & $202(64)$ & $131(63)$ & NS \\
\hline Broncoespasmo & $56(18)$ & $39(19)$ & NS \\
\hline Neumonía & $51(16)$ & $34(16)$ & NS \\
\hline Síndrome coqueluchoide & $6(2)$ & $3(1)$ & NS \\
\hline \multicolumn{4}{|l|}{ Enfermedades crónicas complejas } \\
\hline Al menos una, $\mathrm{n},(\%)$ & $76(24)$ & $68(33)$ & NS \\
\hline Cantidad, med. (RIC) & $0(0-0)$ & $0(0-1)$ & NS \\
\hline Respiratoria, $\mathrm{n}(\%)$ & $53(17)$ & $43(21)$ & NS \\
\hline Cardiovascular, n (\%) & $11(3)$ & $13(6)$ & NS \\
\hline Neuromuscular, n (\%) & $3(1)$ & $2(1)$ & NS \\
\hline Inmunológica, n (\%) & $7(2)$ & $2(1)$ & NS \\
\hline Neurológica, n (\%) & $11(3)$ & $7(3)$ & NS \\
\hline Genética, n $(\%)$ & $5(2)$ & $12(6)$ & NS \\
\hline Metabólica, n (\%) & $0(0)$ & $1(0)$ & NS \\
\hline Prematurez, n (\%) & $10(3)$ & $9(4)$ & NS \\
\hline Gastrointestinal, n (\%) & $2(1)$ & $0(0)$ & NS \\
\hline Otros, n $(\%)$ & $1(0)$ & $3(1)$ & NS \\
\hline \multicolumn{4}{|l|}{ Rescate de gérmenes } \\
\hline Germen identificado, n (\%) & $127(40)$ & $119(57)$ & $<0,001$ \\
\hline Cantidad (n), med. (RIC) & $0(0-1)$ & $1(0-1)$ & 0,011 \\
\hline Rescate múltiple, n (\%) & $1(0)$ & $3(1)$ & NS \\
\hline VSR, $\mathrm{n}(\%)$ & $94(30)$ & $94(45)$ & $<0,001$ \\
\hline Influenza, n (\%) & $9(3)$ & $3(1)$ & NS \\
\hline H. influenzae tipo b, n (\%) & $0(0)$ & $1(0)$ & NS \\
\hline S. pneumoniae, $\mathrm{n}(\%)$ & $0(0)$ & $1(0)$ & NS \\
\hline Adenovirus, $\mathrm{n}(\%)$ & $3(1)$ & $4(2)$ & NS \\
\hline Parainfluenza, n (\%) & $14(4)$ & $16(8)$ & NS \\
\hline Metapneumovirus, n (\%) & $8(3)$ & $2(1)$ & NS \\
\hline B. pertussis, $\mathrm{n}(\%)$ & $1(0)$ & $0(0)$ & NS \\
\hline M. pneumoniae, n (\%) & $0(0)$ & $1(0)$ & NS \\
\hline \multicolumn{4}{|l|}{ Radiografía de tórax } \\
\hline Patológica, n (\%) & $281(89)$ & $200(97)$ & 0,003 \\
\hline Normal, $\mathrm{n}(\%)$ & $34(11)$ & $7(3)$ & 0,004 \\
\hline Hiperinsuflación pulmonar, n (\%) & $138(44)$ & $111(54)$ & 0,035 \\
\hline Infiltrado intersticial, $\mathrm{n}(\%)$ & $89(28)$ & $50(24)$ & NS \\
\hline Infiltrado único / foco de condensación único, n (\%) & $44(14)$ & $21(10)$ & NS \\
\hline \multicolumn{4}{|l|}{ Infiltrados múltiples / } \\
\hline focos de condensación múltiples, n (\%) & $6(2)$ & $11(5)$ & NS \\
\hline Atelectasia, $\mathrm{n}(\%)$ & $4(1)$ & $7(3)$ & NS \\
\hline
\end{tabular}

* Calculado sobre la base de la Sociedad Argentina de Pediatría. Comité Nacional de Crecimiento y Desarrollo. Guía para Evaluación del Crecimiento Físico. SAP: 2013.

CNAF: cánula nasal de alto flujo; Med.: mediana; RIC: rango intercuartil; kg: kilogramos; DE: desvío estándar;

VSR: virus sincicial respiratorio; NS: no significativo. 
La estadía hospitalaria global fue 4,8 días (3,3$6,9)$. La estadía de los pacientes que requirieron CNAF fue de 5,9 $(3,9-8,1)$ días: 5,9 días $(4,9-8,5)$ en ES y $10,4(7,6-26,4)$ en el grupo de FS.

Se observó un descenso de la FR en todos los pacientes desde el inicio del soporte con CNAF; sin embargo, este descenso, en el grupo ES, se mantuvo en el tiempo, mientras que, en el grupo de FS, solo se observó al inicio (Figura 1). El delta
FR intragrupo fue estadísticamente significativo (test $\mathrm{T}$ para medidas repetidas) para el grupo de ES en las cuatro primeras evaluaciones, mientras que, en el grupo de FS, solo lo fue en la primera comparación (Tabla 4). Las comparaciones de FR entre ambos grupos (ES vs. FS) también mostraron diferencias estadísticamente significativas y de magnitud creciente en cada control a partir de la primera hora (Tabla 4).

TABLA 2. Características basales que mostraron diferencias entre los tres hospitales

\begin{tabular}{lcccc}
\hline & $\begin{array}{c}\text { H. Durand } \\
(\mathrm{n}=83, \text { el 15,9\%) }\end{array}$ & $\begin{array}{c}\text { H. Elizalde } \\
(\mathrm{n}=214, \text { el 40,9\%) }\end{array}$ & $\begin{array}{c}\text { H. Gutiérrez } \\
(\mathrm{n}=225, \text { el 43,1 } \%)\end{array}$ & $\mathrm{p}$ \\
\hline M. de I.: bronquiolitis & $52(63)$ & $166(78)$ & $115(51)$ & $<0,001$ \\
M. de I.: broncoespasmo & $16(19)$ & $8(4)$ & $71(32)$ & $<0,001$ \\
ECC (al menos, una), $\mathrm{n}(\%)$ & $19(23)$ & $35(16)$ & $90(40)$ & $<0,001$ \\
ECC respiratoria, $\mathrm{n}(\%)$ & $11(13)$ & $8(4)$ & $77(34)$ & $<0,001$ \\
ECC genética, $\mathrm{n}(\%)$ & $1(1)$ & $5(2)$ & $11(5)$ & 0,059 \\
Rx patológica, $\mathrm{n}(\%)$ & $51(61)$ & $209(98)$ & $221(98)$ & $<0,001$ \\
Rx: hiperinsuflación pulmonar, $\mathrm{n}(\%)$ & $25(30)$ & $100(47)$ & $124(55)$ & $<0,001$ \\
\hline
\end{tabular}

${ }^{*}$ M. de I.: motivo de ingreso; ECC: enfermedades crónicas complejas; Rx: radiografía de tórax.

TABLA 3. Variables fisiológicas de los pacientes que recibieron cánula nasal de alto flujo según el rango etario

\begin{tabular}{lcc}
\hline Variable & $<=6$ meses $(\mathrm{n}=102)$ & $>6$ meses $(\mathrm{n}=91)$ \\
\hline $\mathrm{FR}$, ciclos $/$ min (media, IC 95\%) & $63(61-65)$ & $63(60-65)$ \\
$\mathrm{FC}$, ciclos / min (media, IC 95\%) & $158(155-162)$ & $154(150-159)$ \\
$\mathrm{SaO}_{2}, \%$ (mediana, RIC) & $96(90-98)$ & $96(92-98)$ \\
\hline
\end{tabular}

FR: frecuencia respiratoria; FC: frecuencia cardíaca; IC: intervalo de confianza; $\mathrm{SaO}_{2}$ : saturación arterial de oxígeno; RIC: rango intercuartil.

FIGURA 1. Evolución de parámetros fisiológicos en pacientes con éxito y fracaso del soporte

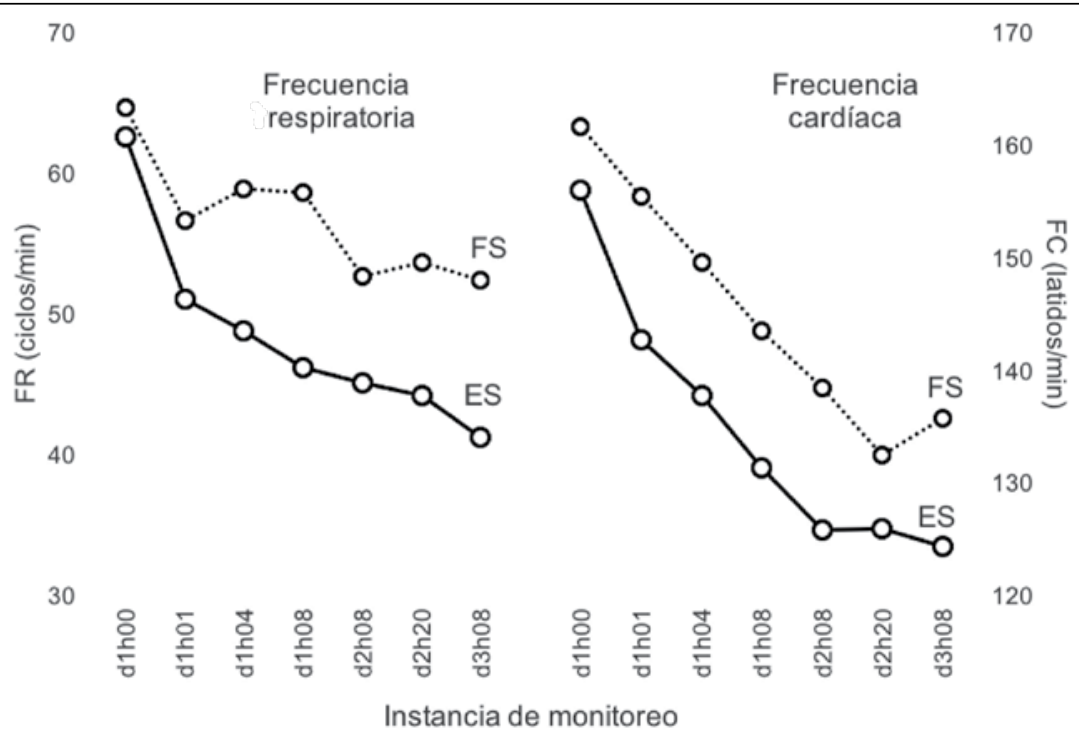

ES: éxito de soporte; FS: fracaso de soporte; FR: frecuencia respiratoria; FC: frecuencia cardíaca.

d1h00: día 1 hora 00 y así sucesivamente. 


\section{Incremento del tratamiento}

Solo 19 de 207 con CNAF (el 8,7 \%) presentaron FS (ingreso a la UCIP): 2 continuaron con CNAF y 8 recibieron VNI (exitosa en 7 casos). La duración de VNI (mediana, RIC) fue 43 horas (33-72) (Figura 2). 10 pacientes requirieron VMI, lo que representó el 52,6\% de FS, el 4,8\% de CNAF y el 1,9\% del total de pacientes admitidos. La duración de la VMI fue 10 días (5-13). La estadía en la UCIP dependió de las estrategias implementadas: aquellos con VMI como único soporte ( $\mathrm{n}=3$ ) permanecieron 10,5 días (5-16); los que continuaron con CNAF y recibieron VMI $(\mathrm{n}=$ 6) estuvieron 13 días (10-16); y el único que recibió VNI y VMI, 15,5 días. No se registró ningún fallecido en toda la muestra.

\section{Seguridad}

No se presentaron, en esta experiencia, complicaciones asociadas al uso de CNAF. Los pacientes pudieron alimentarse por vía oral o sonda nasogástrica sin registrarse eventos adversos asociados.

TABLA 4. Evolución de la frecuencia respiratoria entre pacientes con éxito y fracaso del soporte

\begin{tabular}{|c|c|c|c|c|c|c|c|c|c|c|}
\hline \multirow[b]{2}{*}{ Instancia } & \multicolumn{4}{|c|}{ Éxito } & \multicolumn{4}{|c|}{ Fracaso } & \multicolumn{2}{|c|}{ ES vs. FS } \\
\hline & $\mathbf{n}$ & $\mathbf{F R}^{*}$ & Dif. intra-ES & p\# & $\mathbf{n}$ & $\mathbf{F R}^{*}$ & Dif. intra-FS & p\# & Dif. entre & p\$ \\
\hline Presoporte & 189 & $63(11,0)$ & & - & 18 & $65(9,1)$ & & & $-2,1$ & 0,3696 \\
\hline 1 hora & 187 & $51(10,9)$ & 10,8 & $<0,001$ & 18 & $57(10,4)$ & 7,9 & 0,002 & $-5,6$ & 0,0403 \\
\hline 4 horas & 178 & $48,7(12,1)$ & 2,5 & 0,003 & 17 & $59(12,2)$ & $-1,2$ & 0,639 & $-10,1$ & $<0,001$ \\
\hline 8 horas & 179 & $46,1(11,7)$ & 2,0 & 0,023 & 14 & $59(10,7)$ & $-2,0$ & 0,496 & $-12,5$ & $<0,001$ \\
\hline 8:00, día 1 & 178 & $45(10,6)$ & 0,6 & - & 8 & $53(10,5)$ & 0,0 & - & $-8,1$ & \\
\hline 20:00, día 1 & 171 & $44,1(10,9)$ & 1,8 & - & 8 & $54(13,2)$ & $-4,7$ & - & & \\
\hline 8:00, día 2 & 169 & $41,2(10,3)$ & 0,5 & - & 6 & $52(13,4)$ & $-3,0$ & - & & \\
\hline
\end{tabular}

FR: frecuencia respiratoria; Dif. intra-ES: diferencia intra grupo éxito del soporte;

Dif. intra-FS: diferencia intra grupo fracaso del soporte; Dif. entre: diferencia entre grupos de éxito vs. fracaso de soporte.

* media y desvío estándar.

$\mathrm{p \#}$ : test T para muestras pareadas.

$\mathrm{p} \$$ : test $\mathrm{T}$ para muestras independientes.

FiguRa 2. Transición entre métodos de soporte en pacientes con fracaso del soporte

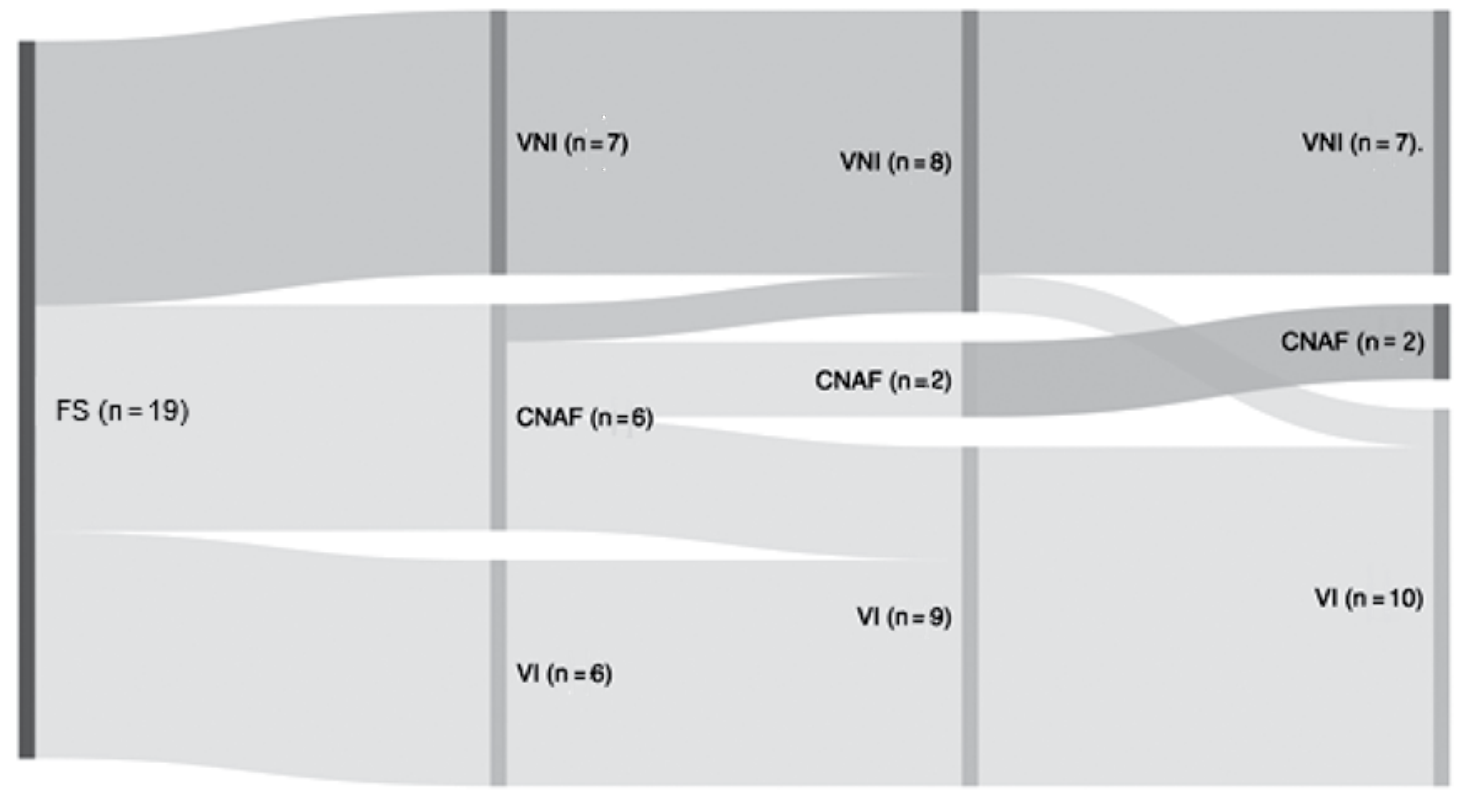

FS: falla del soporte; VNI: ventilación no invasiva; VI: ventilación invasiva; CNAF: cánula nasal de alto flujo. 


\section{DISCUSIÓN}

En esta muestra de pacientes con IRAB internados en salas generales de pediatría en las que se implementó el uso protocolizado de CNAF, se observó que fue seguro. La mejoría sostenida de la FR después de la primera hora de soporte con CNAF es un claro factor pronóstico de éxito del soporte con CNAF.

Se observó un ingreso a la UCIP del 3,6 \%, inferior al reportado para el mismo año en todos los hospitales pediátricos por el Observatorio de Enfermedades Respiratorias Agudas Bajas, dependiente del Ministerio de Salud de la CABA (el 5,1\%). A su vez, esta tasa también fue inferior a la reportada por el mismo organismo para el trienio 2014/6 (el 8,5\%); sin embargo, no se dispone de datos de 2014 / 6 de las unidades participantes en este estudio para poder efectuar una comparación más específica.

En un estudio retrospectivo, Wing y col., ${ }^{6}$ compararon 3 cohortes: la primera cuando no disponían de CNAF (2006); la segunda cuando disponían de CNAF solo en la UCIP (20072008) y la tercera (2009) cuando ya se disponía de CNAF en el Servicio de Emergencias y en la Sala de Pediatría. Incluyeron a 848 pacientes y reportaron una reducción en el uso de ventilación invasiva (VI) del $16 \%$ al $8 \%$. También observaron una asociación entre un inicio más precoz del uso de CNAF y un menor requerimiento de VI (el 7,1 \% vs. el $18 \%$ ), lo que no se observó en nuestro análisis. Posteriormente, en 2013, Gonzales Martínez y col. reportaron un descenso en el ingreso a la UCIP del $12,6 \%$ al $4,6 \%$, al incorporar CNAF en la Sala General en pacientes con bronquiolitis de moderada a grave, ${ }^{19}$ similar a lo reportado por Mayfield y col., (el $31 \%$ vs. el $13 \%$ de ingreso a la UCIP). ${ }^{20}$ En un estudio aleatorizado realizado en un solo centro, Kepreotes y col., al comparar el uso de oxígeno a BF vs. CNAF, encontraron un descenso del FS (el $32 \%$ vs. el $14 \%$ ), similar al reportado por Franklin y col. en un ensayo multicéntrico aleatorizado de reciente publicación, en el que el descenso fue del $23 \%$ al $12 \%$. ${ }^{8,9}$

La FR como predictor de ES encontrada en nuestro análisis coincidió también con lo reportado por otros estudios, en los que un descenso de esta entre la hora y las 4 horas se

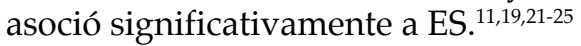

El soporte nutricional enteral fue de particular importancia en la evolución de los pacientes pediátricos. Solo un estudio, hasta el momento, reportó, en 9 pacientes, la seguridad de la alimentación durante el tratamiento con CNAF. ${ }^{24}$ En nuestro análisis, los equipos tratantes no contraindicaron la alimentación enteral por el uso de CNAF. Si bien este dato no fue incluido en el protocolo, la comunicación informal de los investigadores reflejó una conducta homogénea al respecto.

Al igual que lo reportado por los estudios previamente citados, no se observó ningún evento adverso asociado al soporte con CNAF.

Se encontraron diferencias en algunas características basales de los pacientes entre los tres hospitales. Es posible que el universo de pacientes habitualmente asistidos, del cual surgió la población estudiada, presentara diferencias por el perfil de cada institución y su respectivo ámbito de influencia.

La principal limitación del presente estudio fue que, al ser un estudio observacional no controlado, no pudo saberse si el descenso en el ingreso a la UCIP registrado se debió al azar o a la presencia de otros factores más allá del uso de CNAF. Por ello, se decidió continuar con la observación prospectiva durante 2018 para evaluar si este descenso era sostenido.

\section{CONCLUSIÓN}

La implementación protocolizada del uso de CNAF en esta muestra de pacientes con diagnóstico de IRAB ingresados en salas generales de pediatría seleccionadas se mostró como una medida segura, y no se registraron complicaciones relevantes asociadas a su uso. El descenso de la FR en la primera hora de tratamiento se presentó como el factor pronóstico de éxito terapéutico más claro. Los pacientes que tuvieron FS fueron tratados en la UCIP o en la Unidad de Terapia Intermedia, y no hubo una evolución diferente de la habitual.

\section{Agradecimientos}

Hospital Durand: Romina Carosella, Tomas Gilligan, Sabrina Guzmán, Silvia Piccinini, Ana Pota, Viviana Reines, Raúl Ruiz, Sandra Salzberg. Hospital Elizalde: Margarita Jara, Héctor Morales. Hospital Gutiérrez: Mónica Dastugue, Lucila de Luis, Miranda Fernández, Daniela Font, Camila García Laplaza, María Carolina Groisman, Mónica Koch, Florencia Locatelli, María Victoria López, Normando Mascardi, Guadalupe Picón, Lucía Queizan, Natalia Rey, Sofía Rigou. 


\section{REFERENCIAS}

1. Farias JA, Fernández A, Monteverde E, Flores JC, et al. Mechanical ventilation in pediatric intensive care units during the season for acute lower respiratory infection: a multicenter study. Pediatr Crit Care Med. 2012;13(2):158-64.

2. Golubicki A, Gómez Traverso R. Observatorio de Enfermedades Respiratorias Agudas Bajas en Unidades de Internación Pediátricas. Ministerio de Salud de la Ciudad de Buenos Aires; 2015.

3. Comité Nacional de Neumonología, Comité Nacional de Infectología, ComitédeMedicina Interna. Recomendaciones para el manejo de las infecciones respiratorias agudas bajas en menores de 2 años. Resumen ejecutivo. Arch Argent Pediatr. 2015; 113(4):373-4.

4. Dysart K, Miller TL, Wolfson MR, Shaffer TH. Research in high flow therapy: mechanisms of action. Respir Med. 2009; 103(10):1400-5.

5. TenBrinkF,DukeT, EvansJ.High-Flow NasalProng Oxygen Therapy or Nasopharyngeal Continuous Positive Airway Pressure for Children With Moderate-to-Severe Respiratory Distress?* Pediatr Crit Care Med. 2013; 14(7):e326-31.

6. Wing R, James C, Maranda LS, Armsby CC. Use of highflow nasal cannula support in the emergency department reduces the need for intubation in pediatric acute respiratory insufficiency. Pediatr Emerg Care. 2012; 28(11):1117-23.

7. Metge P, Grimaldi C, Hassid S, Thomachot L, et al. Comparison of a high-flow humidified nasal cannula to nasal continuous positive airway pressure in children with acute bronchiolitis: experience in a pediatric intensive care unit. Eur J Pediatr. 2014; 173(7):953-8.

8. Franklin D, Babl FE, Schlapbach LJ, Oakley E, et al. A Randomized Trial of High-Flow Oxygen Therapy in Infants with Bronchiolitis. N Engl J Med. 2018; 378(12):1121-31.

9. Kepreotes E, Whitehead B, Attia J, Oldmeadow C, et al. High-flow warm humidified oxygen versus standard low-flow nasal cannula oxygen for moderate bronchiolitis (HFWHO RCT): an open, phase 4, randomised controlled trial. Lancet. 2017; 389(10072):930-9.

10. Schibler A, Pham TM, Dunster KR, Foster K, et al. Reduced intubation rates for infants after introduction of high-flow nasal prong oxygen delivery. Intensive Care Med. 2011; 37(5):847-52.

11. McKiernan C, Chua LC, Visintainer PF, Allen H. High flow nasal cannulae therapy in infants with bronchiolitis. J Pediatr. 2010; 156(4):634-8.

12. Kawaguchi A, Yasui Y, deCaen A, Garros D. The Clinical Impact of Heated Humidified High-Flow Nasal Cannula on Pediatric Respiratory Distress. Pediatr Crit Care Med. 2017; 18(2):112-9.

13. Tal A, BavilskiC, Yohai D, Bearman JE, et al. Dexamethasone and salbutamol in the treatment of acute wheezing in infants. Pediatrics. 1983;71(1):13-8.

14. Feudtner C, Feinstein JA, Zhong W, Hall M, et al. Pediatric complex chronic conditions classification system version 2: updated for ICD-10 and complex medical technology dependence and transplantation. BMC Pediatr. 2014;14:199.

15. Wasserstein RL, Lazar NA. The ASA's Statement on p-Values: Context, Process, and Purpose. Am Stat. 2016; 70(2):129-33.

16. Kruschke JK. What to believe: Bayesian methods for data analysis. Trends Cogn Sci. 2010; 14(7):293-300.

17. Stanton JM. Reasoning with Data: an Introduction to Traditional and Bayesian Statistics Using R. New York: Guilford Press; 2017.

18. Jamil T, Ly A, Morey RD, Love J, et al. Default "Gunel and Dickey" Bayes factors for contingency tables. Behav Res Methods. 2017; 49(2):638-52.

19. González Martínez F, González Sánchez MI, Rodríguez Fernández R. Impacto clínico de la implantación de la ventilación por alto flujo de oxígeno en el tratamiento de la bronquiolitis en una planta de hospitalización pediátrica. An Pediatr. 2013; 78(4):210-5.

20. MayfieldS, Bogossian F, O'Malley L, Schibler A. High-flow nasal cannula oxygen therapy for infants with bronchiolitis: Pilot study. J Paediatr Child Health. 2014; 50(5):373-8.

21. Kallappa C, Hufton M, Millen G, Ninan TK. Use of high flow nasal cannula oxygen (HFNCO) in infants with bronchiolitis on a paediatric ward: a 3-year experience. Arch Dis Child. 2014; 99(8):790-1.

22. Bressan S, Balzani M, Krauss B, Pettenazzo A, et al. Highflow nasal cannula oxygen for bronchiolitis in a pediatric ward: a pilot study. Eur J Pediatr. 2013; 172(12):1649-56.

23. Hough JL, Pham TMT, Schibler A. Physiologic effect of high-flow nasal cannula in infants with bronchiolitis. Pediatr Crit Care Med. 2014; 15(5):e214-9.

24. Milani GP, Plebani AM, Arturi E, Brusa D, et al. Using a high-flow nasal cannula provided superior results to lowflow oxygen delivery in moderate to severe bronchiolitis. Acta Paediatr. 2016; 105(8):e368-72.

25. Kelly GS, Simon HK, Sturm JJ. High-flow nasal cannula use in children with respiratory distress in the emergency department: predicting the need for subsequent intubation. Pediatr Emerg Care. 2013; 29(8):888-92. 


\section{ANEXO \\ USO DE CÁNULA NASAL DE ALTO FLUJO. EXPERIENCIA EN LA CIUDAD AUTÓNOMA DE BUENOS AIRES, 2017 GRUPO DE ESTUDIO DE ENFERMEDAD RESPIRATORIA AGUDA BAJA EN PEDIATRÍA}

\section{Material suplementario}

Plan de trabajo. Los miembros del equipo de salud de las salas de internación general involucradas recibieron un programa de capacitación teórico-práctico dirigido por los responsables de la implementación y dictado por profesionales capacitados en el uso de CNAF. Sobre la base de la evidencia disponible, se decidió utilizar el sistema de CNAF si se observaba fracaso de la oxigenoterapia de BF de acuerdo con los criterios explicitados en el texto original.

Los pacientes ingresados con diagnóstico de IRAB recibieron tratamiento médico según las guías de tratamiento de pacientes con IRAB del Ministerio de Salud de la CABA. El algoritmo de selección del soporte con oxigenoterapia (Anexo 1) fue incluido en estas guías y colocado impreso en un lugar visible en las salas de internación general y en las UCIP participantes. La evaluación de los pacientes y la decisión de iniciar la terapia con CNAF o de escalar tratamiento quedó a cargo del equipo tratante.

La oxigenoterapia convencional se administró por cánula nasal o máscara con flujos de oxígeno menores de $4 \mathrm{l} / \mathrm{min}$, y se buscó mantener el mismo objetivo de $\mathrm{SaO}_{2}(\geq 94 \%)$ por saturometría de pulso. El destete de la oxigenoterapia $\mathrm{BF}$ se llevó a cabo en todo paciente que lograra el objetivo de $\mathrm{SaO}_{2}$ al respirar aire ambiente.

La oxigenoterapia de AF se administró a través del sistema de AF humidificador Airvo 2 de Fisher \& Paykel Healthcare ${ }^{\circledR}$ con cánula Optiflow infant (hasta $201 / \mathrm{m}$ ) o pediátrica (hasta $25 \mathrm{l} / \mathrm{m}$ ). El Airvo 2 era un humidificador con generador de flujo integrado que permitía administrar oxigenoterapia de AF a una temperatura y humedad óptimas a un flujo y $\mathrm{FiO}_{2}$ programados. El generador de flujo integrado posibilitaba entregar flujos de entre 2 y $601 / \mathrm{m}$. El oxígeno se tomaba desde el suministro estándar de pared (no comprimido) y un analizador de oxígeno ultrasónico integrado aseguraba la administración de la $\mathrm{FiO}_{2}$ deseada. Una tubuladura con cable calentador y sensor de temperatura del gas administrado se conectaba al generador de flujo y a la cánula seleccionada. La cánula se seleccionó de acuerdo con el peso del paciente y se evitó ocluir más del $50 \%$ de las narinas. El flujo se programó, inicialmente, a $21 / \mathrm{kg} / \mathrm{min}$. $\mathrm{La} \mathrm{FiO}_{2}$ se programó, en el comienzo, en 0,9 y bajó rápido a menos de 0,6 luego de que el paciente se hubiera estabilizado con una $\mathrm{SaO}_{2}$ objetivo del $94 \%$ en un período no mayor de tres horas. La $\mathrm{FiO}_{2}$ se descendió 0,05 puntos por vez, se ajustó a una $\mathrm{SaO}_{2}$ del $94 \%$ por saturometría de pulso, y se mantuvo el flujo constante en $21 / \mathrm{kg} / \mathrm{min}$. En caso de observar una caída en la $\mathrm{SaO}_{2}$ por debajo del $94 \%$ al descender la $\mathrm{FiO}_{2}$, esta última se incrementó a los niveles previos. El destete de la CNAF se llevó a cabo en todo paciente que pudiera permanecer, al menos, 4 horas con $\mathrm{FiO}_{2}$ de 0,21.

El protocolo no incluyó ninguna especificación sobre la forma de alimentación de los pacientes, que quedó a criterio del equipo tratante.

Se designó un investigador responsable por cada unidad de internación, quien fue el encargado de registrar todos los datos contemplados en los formularios de este protocolo.

Se definió como ES todo caso que requirió solo tratamiento con CNAF, y se consideró FS todo aquel que requirió algún tratamiento adicional: 1) pase a la UCIP o a Terapia Intermedia, 2) modificación del soporte ventilatorio a VMI o VNI.

Recolección de información: los formularios para la recolección de datos fueron diseñados específicamente para este fin por el equipo coordinador del estudio (Anexo 2). La información registrada en los formularios de papel fue luego incorporada en un formulario web diseñado con la herramienta JotForm (www.jotform.com) por el mismo equipo coordinador. El acceso a estos formularios web estuvo resguardado por usuarios y contraseñas individuales, cuya protección quedó bajo responsabilidad de cada investigador principal. Esos datos, una vez cargados, estuvieron disponibles para su revisión y análisis por los coordinadores del estudio, que monitorearon su evolución en tiempo real. En estos formularios, la identificación de cada paciente individual se realizó mediante un código único asignado dentro de cada institución. La vinculación entre el código del paciente y los datos de identificación personal fue resguardada en cada uno de los hospitales participantes, que se comprometieron a no divulgarla, en virtud de cumplir con lo establecido en la Ley 25326 de Protección de Datos Personales. 